\title{
miR-138 suppresses the proliferation of oral squamous cell carcinoma cells by targeting Yes-associated protein 1
}

\author{
RAN XU ${ }^{1 *}$, GUANG ZENG $^{2 *}$, JING GAO $^{3 *}$, YUE REN $^{1}$, ZHE ZHANG $^{1}$, QINGNA ZHANG $^{1}$, \\ JINXIU ZHAO $^{1}$, HONG TAO ${ }^{1}$ and DAXU LI ${ }^{1}$ \\ ${ }^{1}$ Department of Stomatology, The First Affiliated Hospital, Medical School of Xi'an Jiaotong University, Xi'an, \\ Shaanxi 710061; ${ }^{2}$ Department of Plastic and Burn Surgery, Tangdu Hospital, The Fourth Military Medical University, \\ Xi'an, Shaanxi 710038; ${ }^{3}$ State Key Laboratory of Military Stomatology, Department of Prosthodontics, \\ School of Stomatology, The Fourth Military Medical University, Xi'an, Shaanxi 710032, P.R. China
}

Received May 11, 2015; Accepted July 6, 2015

DOI: 10.3892/or.2015.4144

\begin{abstract}
Aberrant microRNA expression has been suggested to be an important event in the pathologies of various types of cancer. MicroRNA-138 (miR-138) has been reported to be frequently downregulated in various types of human cancer, including oral squamous cell carcinoma (OSCC). However, the precise molecular mechanism of miR-138 underlying OSCC remains largely unknown. The aim of the present study was to investigate the expression of miR-138 in OSCC tumor tissues and several OSCC cell lines and validated its interaction with the 3 '-untranslated region (3'-UTR) of Yes-associated protein 1 (YAP1). The results showed that, miR-138 was significantly downregulated in OSCC tumor tissues and cell lines. Overexpression of miR-138 inhibited cell proliferation of OSCC cells whereas the downregulation of miR-138 promoted cell proliferation. A direct interaction between miR-138 and 3'-UTR of YAP1 was validated by dual-luciferase reporter assay. Moreover, overexpression of miR-138 in OSCC cells significantly decreased the expression of YAP1 and downregulation of miR-138 inhibited the expression of YAP1. Specifically, the inhibitory effect of miR-138 on the proliferation of OSCC cells was eliminated by transfection with YAP1 overexpression vectors that did
\end{abstract}

Correspondence to: Dr Hong Tao or Dr Daxu Li, Department of Stomatology, The First Affiliated Hospital, Medical School of Xi'an Jiaotong University, 277 West Yanta Street, Xi'an, Shaanxi 710061, P.R. China

E-mail: taohong116@163.com

E-mail: lidaxuldx@163.com

${ }^{*}$ Contributed equally

Abbreviations: OSCC, oral squamous cell carcinoma; miR-138, microRNA-138; UTR, untranslated region; YAP1, Yes-associated protein 1; mRNA, messenger RNA

Key words: oral squamous cell carcinoma, miR-138, YAP1, cell proliferation not harbor any specific miR-138 binding specific sequences in 3'-UTR. In addition, the miR-138-overexpressing OSCC cells exhibited a low growth rate in the xenograft tumor assay with a decreased expression of YAP1 in tumor tissues. The results suggest that miR-138 is a tumor suppressor miRNA in OSCC through targeting YAP1, which serves as a promising therapeutic target for the treatment of OSCC.

\section{Introduction}

Oral squamous cell carcinoma (OSCC) is one of the life-threatening cancer types worldwide with a high frequency of recurrence and a low survival rate $(1,2)$. Although many efforts have been made to improve the therapeutic methods for the treatment of OSCC, the therapeutic efficacy and the five-year survival rate remain unsatisfactory (3). Therefore, a better understanding of the molecular pathologies underlying OSCC provides novel insight to develop effective therapeutic strategies for OSCC.

In recent years, microRNAs (miRNAs), a small type of non-coding RNAs, have been found to play an important role in many diseases including cancer $(4,5)$. miRNAs are capable of targeting the 3'-untranslated region (3'-UTR) of messenger RNA (mRNA) containing the corresponding complementary sequences that results in mRNA destabilization and degradation, thereby leading to protein translational inhibition (6). Given their ability to modulate gene expression, miRNAs have been found to participate in many cell processes, including cell growth and proliferation, differentiation, apoptosis; thus, miRNAs are involved in the pathologies of various diseases $(7,8)$. Numerous miRNAs have been identified in various studies on cancer including OSCC (9). For instance, Manikandan et al have reported that miR-21, miR-125b-2*, miR-134, miR-155, miR-184 and miR-205 are dysregulated in OSCC and are associated with clinical pathology (10). A retrospective study of miRNA expression in OSCC tumor tissues from early stage patients has demonstrated that miR-375 and miR-214-3p have a predictive value for prognosis (11). Downregulation of miR-329 and miR-410 increased the proliferation and invasion of OSCC cells by targeting and regulating the expression of Wnt-7b (12). Overexpression of mi-125a has been found to 
inhibit the proliferation and invasion of OSCC cells by modulating estrogen-related receptor $\alpha$ (13). Therefore, targeting the pivotal miRNAs in the pathogenesis of OSCC is expected to provide valid therapeutics for the treatment of OSCC.

MicroRNA-138 (miR-138) has been suggested as a tumor-suppressor gene that is frequently downregulated in various types of cancer, including lung (14-16), thyroid (17), liver (18), colorectal (19) and ovarian (20) cancer. Moreover, miR-138 is involved in OSCC. Loss of heterozygosity in miR-138 precursor genes located at the chromosome loci frequently occurs in OSCC $(21,22)$. A decreased expression of miR-138 was observed in the tumor samples from OSCC (10). However, the precise molecular mechanism of miR-138 in regulating OSCC remains poorly understood. Yes-associated protein 1 (YAP1), a critical transcription co-activator of Hippo pathway, has been suggested as a candidate oncogene in human cancer (23-25). Overexpression of YAP1 has been observed in a number of cancer types and is associated with cell proliferation and tumor growth $(26,27)$. In OSCC, YAP1 has been found to be amplified and overexpressed (28), suggesting an important role of YAP1 in OSCC. Of note, we found through bioinformatics analysis that YAP1 was the predicted target gene of miR-138. Therefore, the aim of the present study was to identify and validate whether miR-138 was involved in the development and progression of OSCC by directly targeting and regulating YAP1.

\section{Materials and methods}

Cell lines and animals. OSCC cell lines, including OC3, $\mathrm{KB}$, OEC-M1, HSC3 and SCC-4, were purchased from the Type Culture Collection of the Chinese Academy of Sciences (Shanghai, China) and were cultured according to the manufacturer's instructions. Normal human oral keratinocytes (HOK) were obtained from ScienCell (Carlsbad, CA, USA) and were grown in oral keratinocyte medium according to the manufacturer's instructions. The cells were maintained in a humidified atmosphere containing $5 \% \mathrm{CO}_{2}$ at $37^{\circ} \mathrm{C}$. Five-week-old male BALB/c nude mice (weighing 25-30 g) were purchased from the Laboratory Animal Centre of the Medical School of Xi'an Jiaotong University (Xi'an, China) and were raised and handled according to the guideline of the Institutional Animal Care and Use Committee of Xi'an Jiaotong University.

Collection of clinical samples. Twenty paired adjacent non-tumorous and tumor tissues from patients undergoing surgical resection were frozen in liquid nitrogen. The present study was approved by and was in accordance with the guidelines of Institutional Human Experiment and the Ethics Committee of the First Affiliated Hospital, Medical School of Xi'an Jiaotong University (Xi'an, China).

Reverse transcriptase-quantitative polymerase chain reaction $(R T-q P C R)$. Total RNA was extracted using TRIzol (Invitrogen, Carlsbad, CA, USA) and was used to generate cDNA using M-MLV reverse transcriptase (Clontech, Palo Alto, CA, USA) and One Step PrimeScript miRNA cDNA Synthesis kit (Takara, Dalian, China) for mRNA and miRNAs analysis, respectively, according to the manufacturer's instructions.
The gene expression was detected using SYBR-Green qPCR Master Mix (Thermo Fisher, Shanghai, China). The relative quantification of the gene expression level was compared with the internal referee GAPDH (for mRNA) or U6 SnRNA (for miRNAs) using the $2^{-\Delta \Delta \mathrm{Ct}}$ method.

Cell infection. Lentivirus (LV)-pre-miR-138, LV-anti-mR-138 and their negative controls were purchased from GenePharma (Shanghai, China). For cell infection, the cells were cultured in a normal medium for $24 \mathrm{~h}$ and then cultured in a medium containing Polybrene (Santa Cruz Biotechnology, Inc., Santa Cruz, CA, USA). The cells were then infected with pre-miR-13, LV-anti-mR-138s or their negative controls on a $0.5 \times 10^{5}$ plaque-forming unit overnight. The old medium was discarded, and a new medium without Polybrene was added and incubated overnight. Stable clones were selected with puromycin dihydrochloride (Santa Cruz Biotechnology, Inc.).

Cell proliferation assay. Cell proliferation was detected by 3-(4,5)-dimethylthiahiazol(-z-y1)-3,5-di-phenytetrazoliumromide (MTT) assay. Briefly, the cells infected with LV-miR-138 or LV-anti-miR-138 were seeded in 96-well plates at $1 \times 10^{4}$ cells/well for 24,48 and $72 \mathrm{~h}$. The old medium was then replaced with fresh medium containing MTT [20 $\mu \mathrm{l} /$ well; $5 \mathrm{mg} / \mathrm{ml}$ diluted in phosphate-buffered saline (PBS)]. The cells were continually cultured for another $4 \mathrm{~h}$, the medium was discarded and dimethylsulfoxide (150 $\mu \mathrm{l} /$ well; Sigma, St. Louis, MO, USA) was added to dissolve the formed formazan crystals. The optical density at $490 \mathrm{~nm}$ was then measured with a microtiter plate reader (Thermo Electron Corporation, Vantaa, Finland).

Dual-luciferase reporter assay. The cDNA fragment of 3'-UTR of YAP1 containing the putative binding site of miR-138 was subcloned into a pGL3 luciferase promoter vector (Promega, Madison, WI, USA). The cells firmly expressing pre-miR-138 or anti-miR-138 were transfected with $0.1 \mu \mathrm{g}$ of pGL3-YAP1-3'-UTR and incubated for $48 \mathrm{~h}$. The cells were collected and lysed. The luciferase activities were quantified using the Dual-Luciferase reporter assay kit (Promega) according to the manufacturer's instructions.

Western blot analysis. Proteins from cells or tumor tissues were extracted using a protein extraction kit (Applygen Technologies, Beijing, China). Protein concentrations in different samples were measured using the Bradford method. For protein separation, a total of $50 \mu \mathrm{g}$ of protein was loaded on $12.5 \%$ sodium dodecyl sulfate polyacrylamide gel electrophoresis. The protein was then transferred to a nitrocellulose membrane (Bio-Rad, Hercules, CA, USA), which was blocked with $3.0 \%$ non-fat milk for $1 \mathrm{~h}$ at $37^{\circ} \mathrm{C}$. Primary antibodies were added and incubated at $4^{\circ} \mathrm{C}$ overnight. Horseradish peroxidaseconjugated secondary antibodies (1:2,000; Bioss, Beijing, China) were added and incubated for $1 \mathrm{~h}$ at room temperature. After washing, the immunoreactive protein bands on the membrane were visualized using an enhanced chemiluminescence detection system (Amersham, Little Chalfont, UK). The primary antibodies used in these experiments, including anti-YAP1 and anti-GAPDH antibodies were purchased from Santa Cruz Biotechnology, Inc. 
A

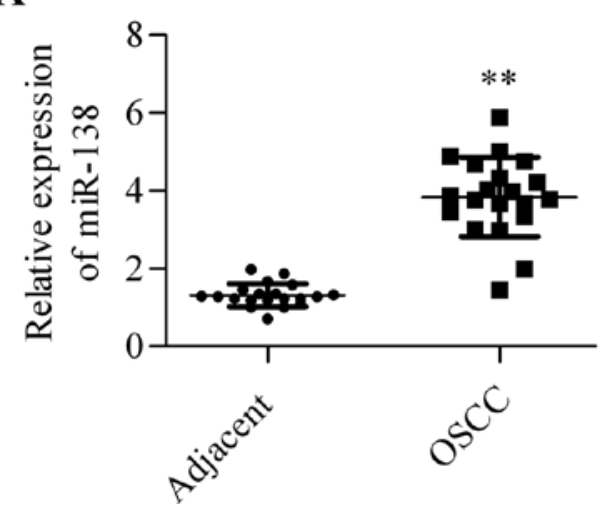

B

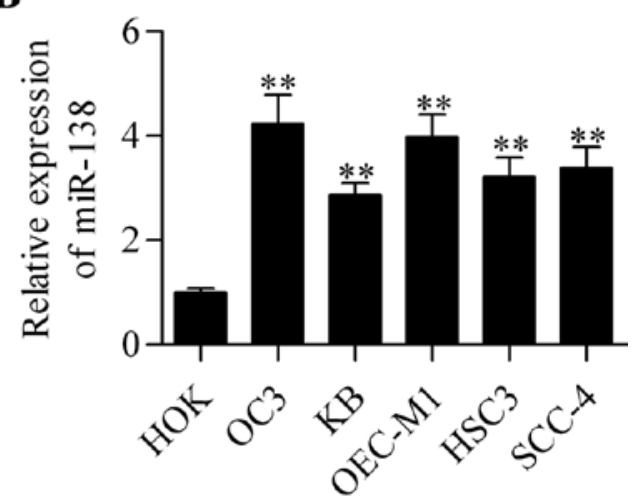

Figure 1. miR-138 was reduced in OSCC. (A) RT-qPCR analysis of miR-138 expression between OSCC and matched adjacent non-tumorous tissues from OSCC patients. $\mathrm{N}=20,{ }^{* *} \mathrm{p}<0.01$ vs. adjacent; (B) RT-qPCR analysis of miR-138 in different cell lines, including OC 3 , KB, OEC-M1, HSC3 and SCC-4 cell lines. HOK cells were used as control. $\mathrm{N}=3,{ }^{* *} \mathrm{p}<0.01$ vs. HOK. OSCC, oral squamous cell carcinoma; HOK, human oral keratinocytes.

Xenograft tumor assay. OEC-M1 cells (1x106 cells) infected with LV-pre-miR-138 or LV-anti-miR-138 diluted in $200 \mu \mathrm{l}$ of PBS were inoculated subcutaneously into the left flank of nude mice. The size of the tumor (length and width) was measured every 3 days by a vernier caliper. The tumor volume was presented as length $\mathrm{x}$ width ${ }^{2} \mathrm{x} \pi / 6$. At the end of the experiment, the tumor was isolated, weighed and extracted for further analysis.

Statistical analysis. Data are presented as means \pm standard deviation and processed using SPSS version 11.5 (SPSS, Inc., Chicago, IL, USA). Statistical differences were obtained by the Student's t-test or one-way analysis of variance followed by Bonferroni post hoc test. Differences were considered statistically significant at $\mathrm{P}<0.05$.

\section{Results}

Downregulated expression of miR-138 is in OSCC tissues and cell lines. To examine the role of miR-138 in OSCC, the expression profile of miR-138 in tumor and adjacent non-tumorous epithelia was quantified by RT-qPCR analysis. Generally, miR-138 was significantly downregulated in OSCC tumor tissues as compared with that in the adjacent non-tumorous tissues (Fig. 1A). In addition, the expression of miR-138 in several different OSCC cell lines was detected. Furthermore, the results showed that miR-138 expression was markedly reduced to different degrees in OC3, KB, OEC-M1, HSC3 and SCC-4 cell lines than that in the control HOK cells (Fig. 1B).

miR-138 suppresses the proliferation of OSCC cells. Considering the downregulated expression of miR-138 in OSCC, we investigated the function of miR-138 in OSCC cells. OSCC cells with miR-138 overexpression or downregulation were generated in OC3 (Fig. 2A) and OEC-M1 (Fig. 2B) cells by infecting with LV-pre-miR-138 or LV-anti-miR-138. MTT assay results showed that miR-138 overexpression significantly decreased the proliferation in OC3 cells, whereas miR-138 downregulation markedly increased OC3 cell proliferation (Fig. 2C). Additionally, similar results were obtained using OEC-M1 cells (Fig. 2D).
YAPl is the target gene of $m i R-138$. To investigate the potential mechanism of the miR-138 regulative role in OSCC, we predicted the target gene of miR-138 by bioinformatics analysis. Notably, we found that the 3'-UTR of YAP1, a candidate oncogene in various types of cancer (29), processed the putative binding sites for miR-138 (Fig. 3A). The wild-type and the mutants of 3'-UTR of YAP1 in the predicted binding sequences were constructed into luciferase reporters to confirm the direct binding between miR-138 and 3'-UTR of YAP1. These reporters were then transfected into HEK293 cells with miR-138 overexpression or downregulation by infection with LV-pre-miR-138 and LV-anti-138. The reporter assay exhibited that miR-138 overexpression significantly inhibited the luciferase activity in wild-type transfected cells, whereas miR-138 downregulation markedly increased the luciferase activity in wild-type transfected cells. Additionally, the aberrant expression of miR-138 had no obvious effect on the luciferase activity in the mutated transfected cells (Fig. 3B).

miR-138 reduces the expression of YAP1 in OSCC cells. To verify the interaction between miR-138 and YAP1, we observed the effect of the ectopic expression of miR-138 on YAP1 expression in OSCC cells. RT-qPCR analysis revealed that miR-138 overexpression significantly decreased the mRNA expression of YAP1 in OC3 (Fig. 4A) and OEC-M1 (Fig. 4B). By contrast, miR-138 inhibition markedly increased the YAP1 mRNA expression in OC3 and OEC-M1 cells. Similar results were obtained in the western blot analysis, which demonstrated that the protein expression of YAP1 was repressed by miR-138 overexpression or promoted by miR-138 downregulation (Fig. 4C and D). To assess whether miR-138 regulated the cell proliferation of OSCC cells by action on YAP1, we transfected YAP1 overexpression vectors, which harbored no specific miR-138 binding specific sequences in 3'-UTR in miR-138-overexpressing cells. The results showed that the overexpression of YAP1 significantly abrogated the inhibitory effect of miR-138 on the cell proliferation of OSCC cells (Fig. 4E and F).

miR-138 represses the xenograft tumor growth in vivo. To confirm the above findings of miR-138, an in vivo xenograft 
A

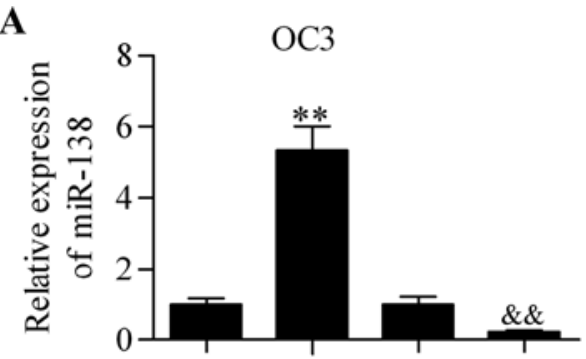

B

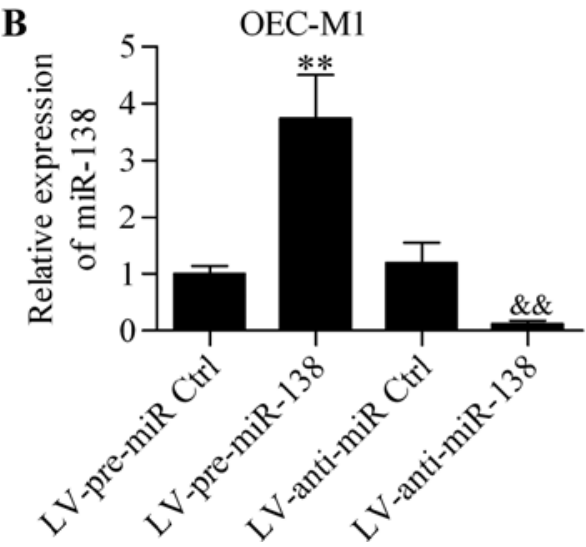

C

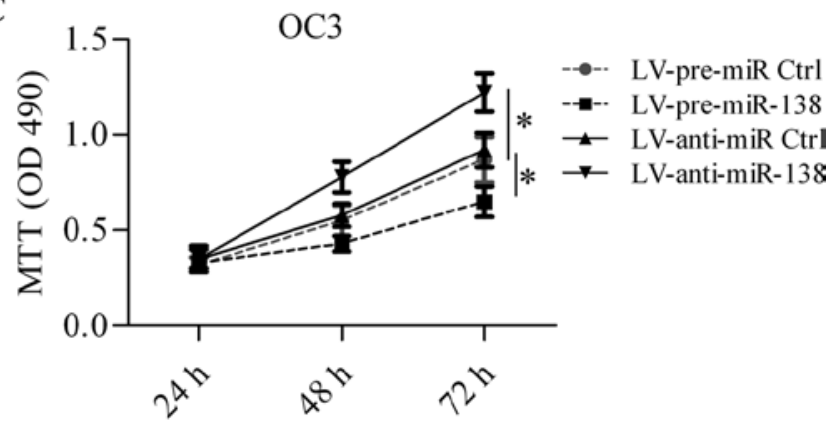

D

OEC-M1

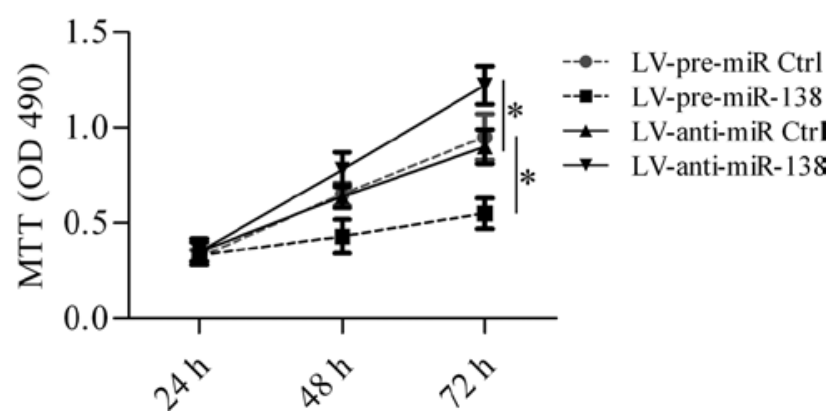

Figure 2. miR-138 inhibits the proliferation of OSCC cells. RT-qPCR analysis of miR-138 expression in (A) OC3 and (B) OEC-M1 cells infected with LV-pre-miR-138 or LV-anti-miR-138. N=3, ${ }^{*}$ p $<0.01$ vs. LV-pre-miR Ctrl; and $\mathrm{p}<0.01$ vs. LV-anti-miR Ctrl. The cell proliferation of (C) OC3 and (D) OEC-M1 cells with miR-138 overexpression or downregulation was examined by an MTT assay at 24,48 and $72 \mathrm{~h}, \mathrm{~N}=6,{ }^{*} \mathrm{p}<0.05$. OSCC, oral squamous cell carcinoma.
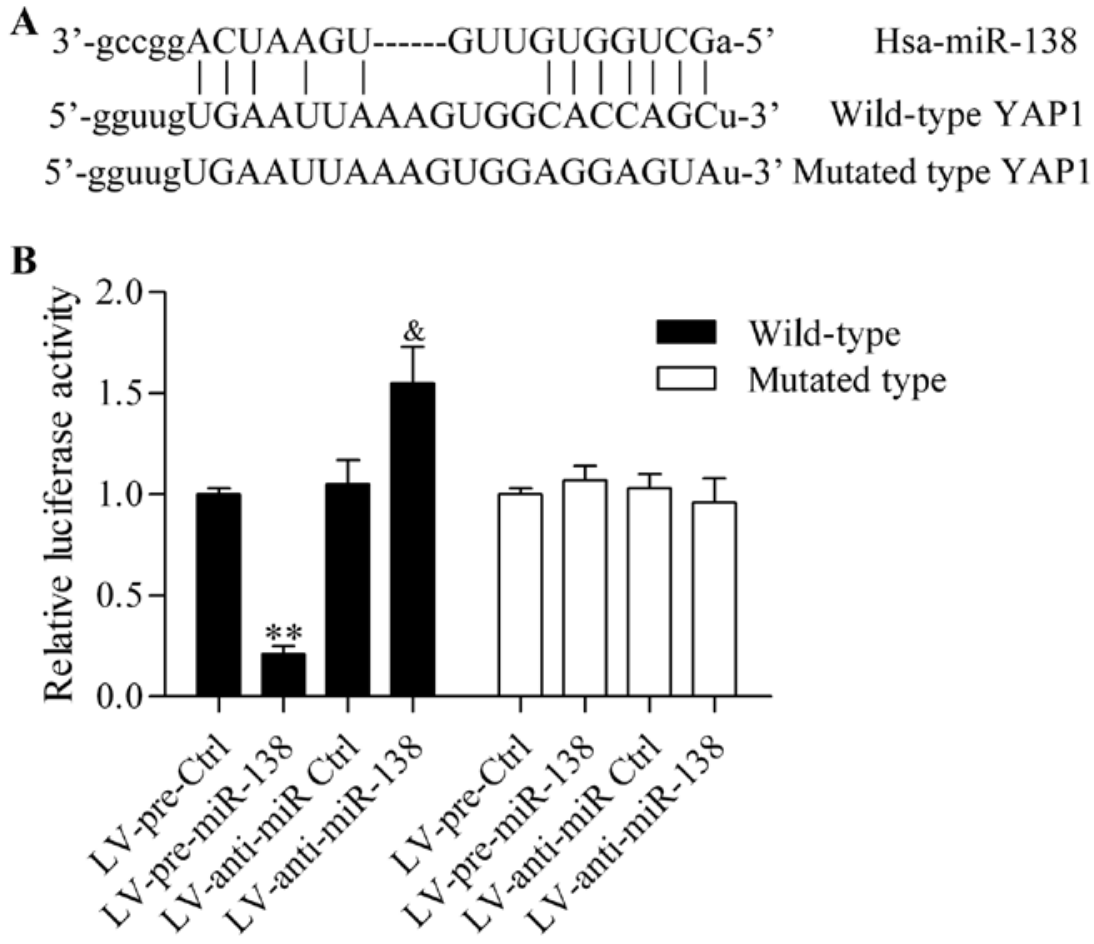

Figure 3. miR-318 targets the 3'-UTR of YAP1. (A) Schedule of binding sites between miR-138 and 3'-UTR of YAP1. (B) Luciferase activity assay detecting the direct binding between miR-138 and 3'-UTR of YAP1. The wild-type or mutated 3'-UTR of YAP1 containing binding sites in luciferase reporters was transfected into HEK293 cells with miR-138 overexpression or silencing. N=3, ${ }^{* *}$ p $<0.01$ vs. LV-pre-miR Ctrl; and p<0.05 vs. LV-anti-miR Ctrl. 3'-UTR, 3'-untranslated region; YAP1, Yes-associated protein 1.

assay was performed using overexpressed stable OC3 cells to test the inhibitory effect of miR-138 on tumor growth in vivo. Compared with the control group, the overexpression of miR-138 significantly reduced the average tumor weight (Fig. 5A) and tumor volume (Fig. 5B) in nude mice subcutaneously injected with OC3 cells. Moreover, the expression of miR-138 and YAP1 in harvested tumor tissues were detected. The results showed that miR-138 was significantly 
A

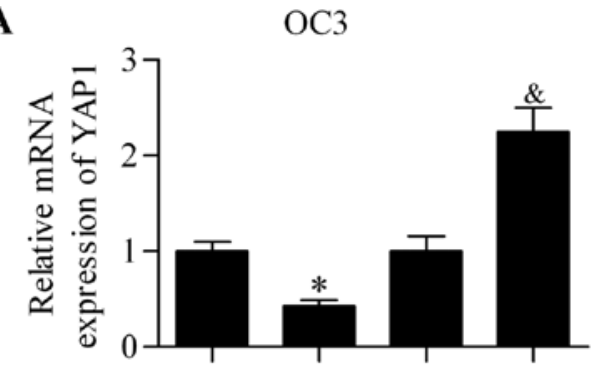

C

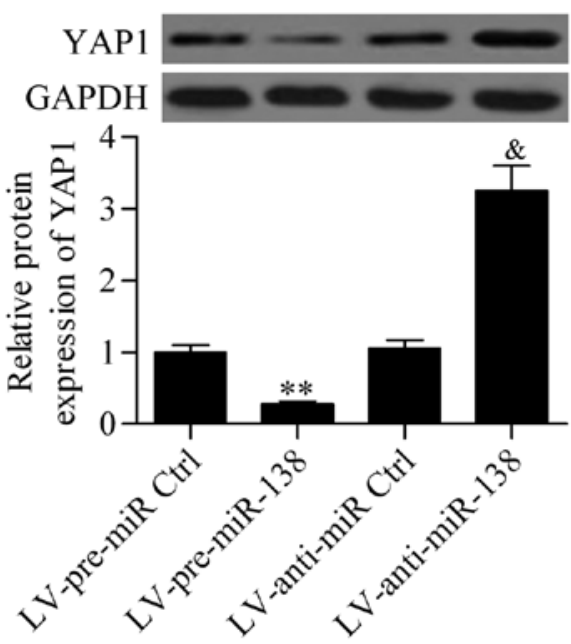

E

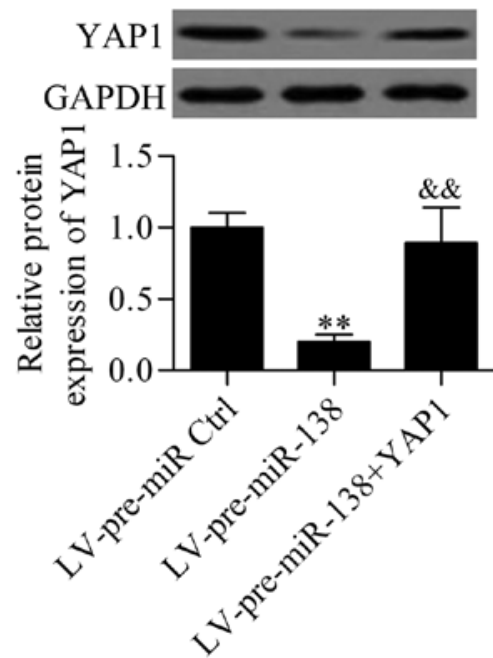

B

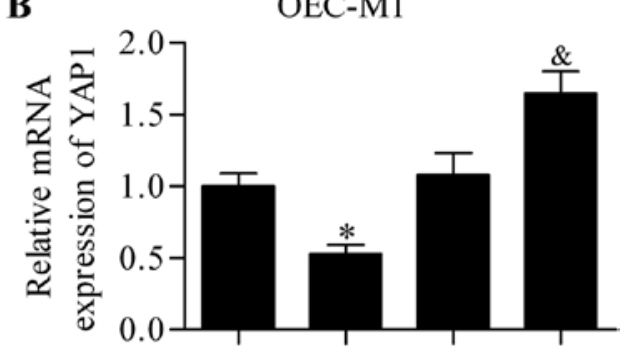

D
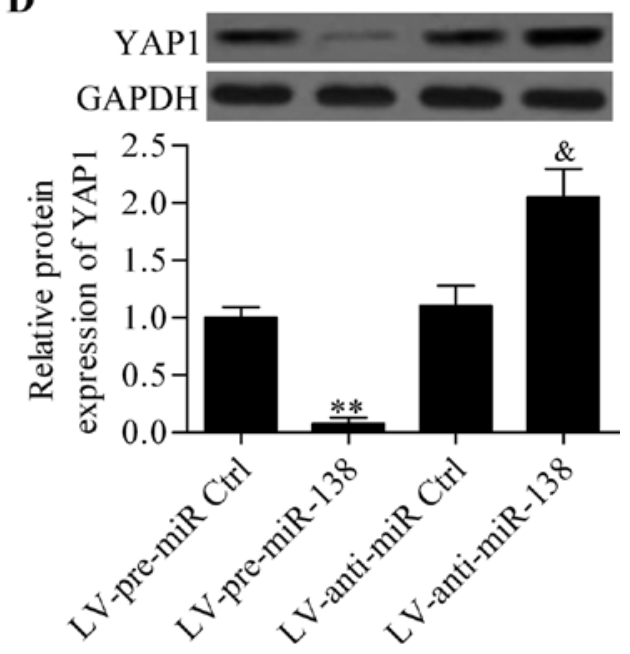

F

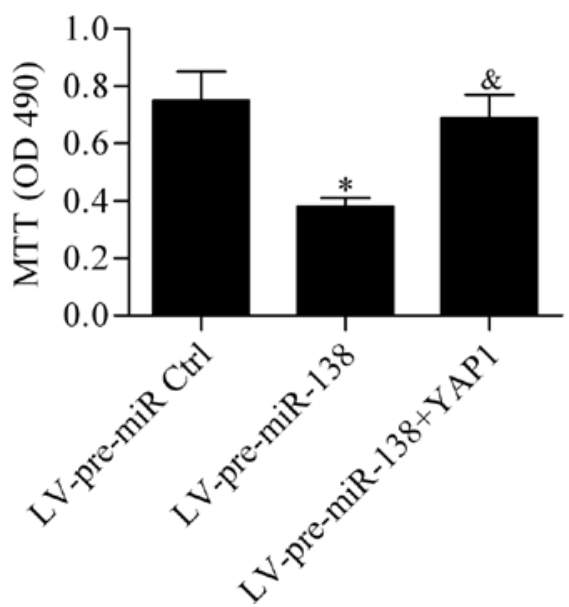

Figure 4. miR-138 suppresses the expression of YAP1. RT-qPCR analysis of YAP1 mRNA expression in (A) OC3 and (B) OEC-M1 cells infected with LV-pre-miR-138 or LV-anti-miR-138. N=3, ${ }^{*}$ p $<0.05$ vs. LV-pre-miR Ctrl; ${ }^{*} \mathrm{p}<0.05$ vs. LV-anti-miR Ctrl. Western blot analysis of YAP1 protein expression in (C) OC3 and (D) OEC-M1 cells. Relative protein expression level was quantified using Image-Pro Plus 6.0 software and normalized to GAPDH. N=3, ${ }^{* *}<<0.01$ vs. LV-pre-miR Ctrl; ${ }^{\circledR}$ p $<0.05$ vs. LV-anti-miR Ctrl. (E) Western blot analysis of YAP1 protein expression in different groups. N=3, ${ }^{* *} p<0.01$ vs. LV-pre-miR $\mathrm{Ctrl}$; ${ }^{\&} \mathrm{p} p<0.01$ vs. LV-pre-miR-138. (E) Cell proliferation was detected by MTT assay in different groups. N=6, ${ }^{*} \mathrm{p}<0.05$ vs. LV-pre-miR Ctrl; ${ }^{\circ} \mathrm{p}<0.05$ vs. LV-pre-miR-138. YAP1, Yes-associated protein 1.

increased in the tumors derived from miR-138-overexpressing OC3 cells (Fig. 5C), whereas the protein expression level of YAP1 was significantly decreased (Fig. 5D).

\section{Discussion}

In the present study, we have reported that a decreased expression of miR-138 in OSCC tumor tissues and OSCC cells occurred frequently, leading to the overexpression of the oncogene YAP1. Consistent with previous findings (30), our results confirm that miR-138 was dysregulated in OSCC. Moreover, to the best of our knowledge, for the first time, we have delineated a direct interaction of miR-138 and YAP1 that may be involved in the regulation of OSCC.

The important role of miR-138 in human types of cancer has been reported. Studies have shown that miR-138 is 
A

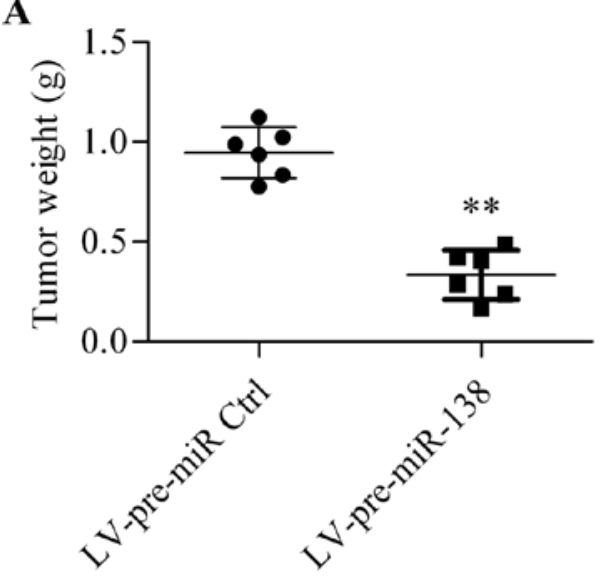

C

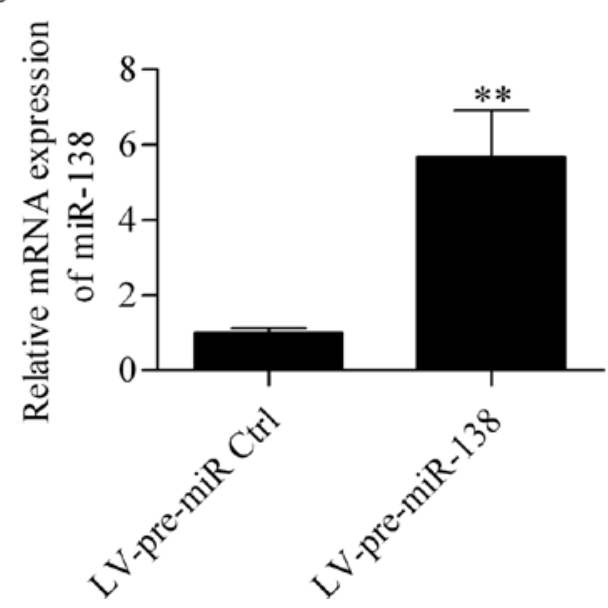

B

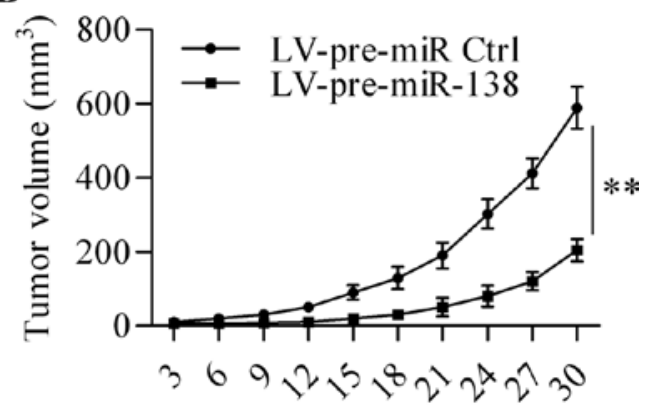

Days after tumor implantation

D

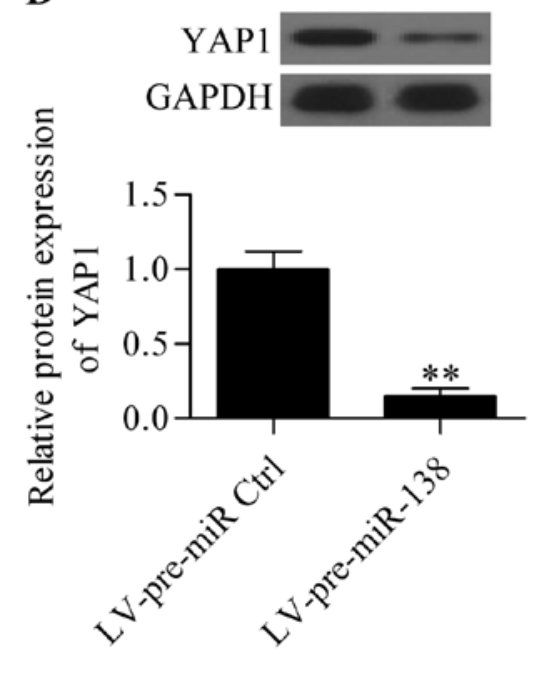

Figure 5. miR-138 represses tumor growth of OSCC in vivo. (A) Tumor weight of each group. At the end of the experiment, mice were sacrificed and the tumors were isolated and weighed in each mouse. $\mathrm{N}=6,{ }^{* *} \mathrm{p}<0.01$. (B) Tumor volumes were measured during the tumor growth process. The tumor volume was measured every 3 days and presented as length $\mathrm{x}$ width ${ }^{2} \mathrm{x} \pi / 6 . \mathrm{N}=6,{ }^{*} \mathrm{p}<0.01$. (C) RT-qPCR analysis of miR-138 expression in tumors derived from miR-138-overexpressing OC 3 cells. $\mathrm{N}=3,{ }^{* *} \mathrm{p}<0.01$. (D) Western blot analysis of YAP1 protein expression in tumors of each group. $\mathrm{N}=3$, ${ }^{* *} \mathrm{p}<0.01$. OSCC, oral squamous cell carcinoma; YAP1, Yes-associated protein 1.

frequently decreased in non-small cell lung cancer tissues and cells and that the overexpression of miR-138 inhibited cancer cell growth in vitro and in vivo by targeting the zeste homolog 2 enhancer (15). More recently, miR-138 has been recognized as a potential prognostic factor for overall survival in non-small cell lung cancer patients by regulating 3-phosphoinositide-dependent protein kinase-1 (31). In addition, Ye et al reported that miR-138 suppressed lung cancer cell proliferation by inhibiting 3-phosphoinositidedependent protein kinase-1 (16). In human anaplastic thyroid carcinoma cells, the decreased miR-138 was associated with increased human telomerase reverse transcriptase protein (17). Furthermore, miR-138 was demonstrated to be involved in regulating hepatocellular and colorectal cancer by targeting cyclin D3 and the Twist basic helix-loop-helix transcription factor 2 gene $(18,19)$. In head and neck squamous cell carcinoma cell lines, the overexpression of miR-138 inhibited cell invasion and increased cell cycle arrest and apoptosis, whereas knockdown of miR-138 exhibited the opposite effect (30). Jiang et al reported that miR-138 inhibited cell migration and invasion in tongue squamous cell carcinoma through the downregulation of RhoC and ROCK2, which constitute the pivotal genes of the Rho GTPase pathway (32). Additionally, $\mathrm{G}$ protein $\alpha$ inhibiting activity polypeptide 2 was validated as a direct target gene of miR-138 in tongue squamous cell carcinoma (33). In the present study, we have demonstrated that miR-138 directly regulates YAP1 in OSCC cells. We report that the overexpression of miR-138 effectively decreased the mRNA and protein expression in OSCC cells as well as cell proliferation and tumor growth. By contrast, the downregulation of miR-138 exhibited the opposite effect.

YAP1 has been proposed as an oncogene in many human types of cancers (23-25). Overexpression of YAP1 has been suggested to be associated with tumor cell proliferation and growth, cell metastasis and poor prognosis of cancer cells $(26,27,34,35)$. In OSCC, YAP1 has been found to be amplified and overexpressed (28), suggesting the important role of YAP1 in OSCC. Moreover, recurrent amplifications of YAP1 have been reported in gingivo-buccal OSCC (36). Zhang et al reported that YAP1 is overexpressed in OSCC cell lines and promotes cell proliferation by activating Fos-related activator 1 (37). Thus, the therapeutic targeting of YAP1 is a promising method for repressing cancer. Notably, multiple miRNAs have been reported to regulate YAP1 expression in human types of cancers. Recent findings have shown that targeting and inhibiting YAP1 by miR-141 suppress cell growth and colony formation and elevate cell apoptosis in pancreatic ductal adenocarcinoma cells (38). Zhang et al 
have delineated that YAP1 is highly expressed in pancreatic progenitor cells, and miR-375 targets the 3'-UTR of YAP1 and decreases its mRNA and protein expression (39). miR-375 was also reported to regulate YAP1 expression in avian leukosis (40). In colorectal cancer, miR-375 overexpression accelerates cell apoptosis by directly targeting YAP1 and its downstream anti-apoptotic targets (41). We have demonstrated that miR-138, a tumor-suppressor gene, also effectively targets YAP1 and is capable of suppressing YAP1, mRNA and protein expression levels in OSCC cells. The downregulated expression of miR-138 in OSCC tissues and cell lines may account for the increased expression of YAP1. Furthermore, we found that overexpression of miR-138 significantly inhibited cell proliferation and tumor growth in vitro and in vivo. Our results demonstrate a direct interaction between miR-138 and YAP1 and their critical role in OSCC, and confirm that the targeting and inhibition of YAP1 by miRNA is a feasible therapeutic treatment of various types of cancer.

In the present study, we have identified a significant role of miR-138 in OSCC that suppressed cell proliferation and growth of OSCC by targeting and inhibiting YAP1 expression. Our results suggest that miR-138 is a tumor suppressor miRNA in OSCC by targeting and inhibiting YAP1, which serves as a promising therapeutic target for the treatment of OSCC.

\section{References}

1. Warnakulasuriya S: Global epidemiology of oral and oropharyngeal cancer. Oral Oncol 45: 309-316, 2009.

2. Siegel R, Ma J, Zou Z and Jemal A: Cancer statistics, 2014. CA Cancer J Clin 64: 9-29, 2014.

3. Haddad RI and Shin DM: Recent advances in head and neck cancer. N Engl J Med 359: 1143-1154, 2008.

4. Li Y and Kowdley KV: MicroRNAs in common human diseases. Genomics Proteomics Bioinformatics 10: 246-253, 2012.

5. Jansson MD and Lund AH: MicroRNA and cancer. Mol Oncol 6: 590-610, 2012

6. Bartel DP: MicroRNAs: Genomics, biogenesis, mechanism, and function. Cell 116: 281-297, 2004

7. Mendell JT and Olson EN: MicroRNAs in stress signaling and human disease. Cell 148: 1172-1187, 2012.

8. Ranganathan K and Sivasankar V: MicroRNAs - Biology and clinical applications. J Oral Maxillofac Pathol 18: 229-234, 2014

9. Gomes CC, de Sousa SF and Gomez RS: MicroRNAs: Small molecules with a potentially role in oral squamous cell carcinoma. Curr Pharm Des 19: 1285-1291, 2013

10. Manikandan M, Deva Magendhra Rao AK, Rajkumar KS, Rajaraman R and Munirajan AK: Altered levels of miR-21, miR-125b-2*, miR-138, miR-155, miR-184, and miR-205 in oral squamous cell carcinoma and association with clinicopathological characteristics. J Oral Pathol Med: Dec 8, 2014 (Epub ahead of print). doi: 10.1111/jop.12300.

11. Yoon AJ, Wang S, Shen J, Robine N, Philipone E, Oster MW, Nam A and Santella RM: Prognostic value of miR-375 and miR-214-3p in early stage oral squamous cell carcinoma. Am J Transl Res 6: 580-592, 2014.

12. Shiah SG, Hsiao JR, Chang WM, Chen YW, Jin YT, Wong TY, Huang JS, Tsai ST, Hsu YM, Chou ST, et al: Downregulated miR329 and miR410 promote the proliferation and invasion of oral squamous cell carcinoma by targeting Wnt-7b. Cancer Res 74: 7560-7572, 2014.

13. Tiwari A, Shivananda S, Gopinath KS and Kumar A: MicroRNA-125a reduces proliferation and invasion of oral squamous cell carcinoma cells by targeting estrogen-related receptor $\alpha$ : Implications for cancer therapeutics. J Biol Chem 289: 32276-32290, 2014.

14. Seike M, Goto A, Okano T, Bowman ED, Schetter AJ, Horikawa I, Mathe EA, Jen J, Yang P, Sugimura H, et al: MiR-21 is an EGFR-regulated anti-apoptotic factor in lung cancer in never-smokers. Proc Natl Acad Sci USA 106: 12085-12090, 2009.
15. Zhang $\mathrm{H}$, Zhang $\mathrm{H}$, Zhao M, Lv Z, Zhang $\mathrm{X}$, Qin $\mathrm{X}$, Wang $\mathrm{H}$, Wang S, Su J, Lv X, et al: MiR-138 inhibits tumor growth through repression of EZH2 in non-small cell lung cancer. Cell Physiol Biochem 31: 56-65, 2013.

16. Ye XW, Yu H, Jin YK, Jing XT, Xu M, Wan ZF and Zhang XY: miR-138 inhibits proliferation by targeting 3-phosphoinositidedependent protein kinase-1 in non-small cell lung cancer cells. Clin Respir J 9: 27-33, 2015.

17. Mitomo S, Maesawa C, Ogasawara S, Iwaya T, Shibazaki M, Yashima-Abo A, Kotani K, Oikawa H, Sakurai E, Izutsu N, et al: Downregulation of miR-138 is associated with overexpression of human telomerase reverse transcriptase protein in human anaplastic thyroid carcinoma cell lines. Cancer Sci 99: 280-286, 2008.

18. Wang W, Zhao LJ, Tan YX, Ren H and Qi ZT: MiR-138 induces cell cycle arrest by targeting cyclin D3 in hepatocellular carcinoma. Carcinogenesis 33: 1113-1120, 2012.

19. Long L, Huang G, Zhu H, Guo Y, Liu Y and Huo J: Downregulation of miR-138 promotes colorectal cancer metastasis via directly targeting TWIST2. J Transl Med 11: 275, 2013.

20. Chen P, Zeng M, Zhao Y and Fang X: Upregulation of Limk1 caused by microRNA-138 loss aggravates the metastasis of ovarian cancer by activation of Limk1/cofilin signaling. Oncol Rep 32: 2070-2076, 2014.

21. Hogg RP, Honorio S, Martinez A, Agathanggelou A, Dallol A, Fullwood P, Weichselbaum R, Kuo MJ, Maher ER and Latif F: Frequent $3 p$ allele loss and epigenetic inactivation of the RASSF1A tumour suppressor gene from region 3p21.3 in head and neck squamous cell carcinoma. Eur J Cancer 38: 1585-1592, 2002.

22. Piccinin S, Gasparotto D, Vukosavljevic T, Barzan L, Sulfaro S, Maestro R and Boiocchi M: Microsatellite instability in squamous cell carcinomas of the head and neck related to field cancerization phenomena. Br J Cancer 78: 1147-1151, 1998.

23. Harvey KF, Pfleger CM and Hariharan IK: The Drosophila Mst ortholog, hippo, restricts growth and cell proliferation and promotes apoptosis. Cell 114: 457-467, 2003.

24. Edgar BA: From cell structure to transcription: Hippo forges a new path. Cell 124: 267-273, 2006.

25. Overholtzer M, Zhang J, Smolen GA, Muir B, Li W, Sgroi DC, Deng CX, Brugge JS and Haber DA: Transforming properties of $Y A P$, a candidate oncogene on the chromosome 11q22 amplicon. Proc Natl Acad Sci USA 103: 12405-12410, 2006.

26. Zhao B, Wei X, Li W, Udan RS, Yang Q, Kim J, Xie J, Ikenoue T, $\mathrm{Yu} \mathrm{J}, \mathrm{Li} \mathrm{L}$, et al: Inactivation of YAP oncoprotein by the Hippo pathway is involved in cell contact inhibition and tissue growth control. Genes Dev 21: 2747-2761, 2007.

27. Hamaratoglu F, Gajewski K, Sansores-Garcia L, Morrison C, Tao C and Halder G: The Hippo tumor-suppressor pathway regulates apical-domain size in parallel to tissue growth. J Cell Sci 122: 2351-2359, 2009.

28. Snijders AM, Schmidt BL, Fridlyand J, Dekker N, Pinkel D, Jordan RC and Albertson DG: Rare amplicons implicate frequent deregulation of cell fate specification pathways in oral squamous cell carcinoma. Oncogene 24: 4232-4242, 2005.

29. Seton-Rogers S: Oncogenes: All eyes on YAP1. Nat Rev Cancer 14: 514-515, 2014.

30. Liu X, Jiang L, Wang A, Yu J, Shi F and Zhou X: MicroRNA-138 suppresses invasion and promotes apoptosis in head and neck squamous cell carcinoma cell lines. Cancer Lett 286: 217-222, 2009.

31. Han L, Zhang G, Zhang N, Li H, Liu Y, Fu A and Zheng Y: Prognostic potential of microRNA-138 and its target mRNA PDK1 in sera for patients with non-small cell lung cancer. Med Oncol 31: 129, 2014.

32. Jiang L, Liu X, Kolokythas A, Yu J, Wang A, Heidbreder CE, Shi F and Zhou X: Downregulation of the Rho GTPase signaling pathway is involved in the microRNA-138-mediated inhibition of cell migration and invasion in tongue squamous cell carcinoma. Int J Cancer 127: 505-512, 2010.

33. Jiang L, Dai Y, Liu X, Wang C, Wang A, Chen Z, Heidbreder CE, Kolokythas A and Zhou X: Identification and experimental validation of $\mathrm{G}$ protein alpha inhibiting activity polypeptide 2 (GNAI2) as a microRNA-138 target in tongue squamous cell carcinoma. Hum Genet 129: 189-197, 2011.

34. Liu R, Huang S, Lei Y, Zhang T, Wang K, Liu B, Nice EC, Xiang R, Xie K, Li J, et al: FGF8 promotes colorectal cancer growth and metastasis by activating YAP1. Oncotarget 6: 935-952, 2015. 
35. Lee KW, Lee SS, Kim SB, Sohn BH, Lee HS, Jang HJ, Park YY, Kopetz S, Kim SS, Oh SC, et al: Significant association of oncogene YAP1 with poor prognosis and cetuximab resistance in colorectal cancer patients. Clin Cancer Res 21: 357-364, 2015.

36. India Project Team of the International Cancer Genome Consortium: Mutational landscape of gingivo-buccal oral squamous cell carcinoma reveals new recurrently-mutated genes and molecular subgroups. Nat Commun 4: 2873, 2013.

37. Zhang L, Ye DX, Pan HY, Wei KJ, Wang LZ, Wang XD, Shen GF and Zhang ZY: Yes-associated protein promotes cell proliferation by activating Fos Related Activator-1 in oral squamous cell carcinoma. Oral Oncol 47: 693-697, 2011.

38. Zhu ZM, Xu YF, Su QJ, Du JD, Tan XL, Tu YL, Tan JW and Jiao HB: Prognostic significance of microRNA-141 expression and its tumor suppressor function in human pancreatic ductal adenocarcinoma. Mol Cell Biochem 388: 39-49, 2014.
39. Zhang ZW, Men T, Feng RC, Li YC, Zhou D and Teng CB: miR-375 inhibits proliferation of mouse pancreatic progenitor cells by targeting YAP1. Cell Physiol Biochem 32: 1808-1817, 2013.

40. Li H, Shang H, Shu D, Zhang H, Ji J, Sun B, Li H and Xie Q: gga-miR-375 plays a key role in tumorigenesis post subgroup $\mathrm{J}$ avian leukosis virus infection. PLoS One 9: e90878, 2014.

41. Christensen LL, Holm A, Rantala J, Kallioniemi O, Rasmussen MH, Ostenfeld MS, Dagnaes-Hansen F, Øster B, Schepeler T, Tobiasen H, et al: Functional screening identifies miRNAs influencing apoptosis and proliferation in colorectal cancer. PLoS One 9: e96767, 2014. 